\title{
Distributed photothermal measurements of gas presence along holey optical fibers
}

\author{
Andres Garcia-Ruiz*a , Juan Pastor-Graells ${ }^{\mathrm{a}}$, Hugo F. Martins ${ }^{\mathrm{b}}$, Kenny Hey Tow ${ }^{\mathrm{c}}$, Luc Thévenaz ${ }^{\mathrm{c}}$, \\ Sonia Martin-Lopez ${ }^{\mathrm{a}}$, Miguel Gonzalez-Herraez ${ }^{\mathrm{a}}$ \\ ${ }^{a}$ Dpto. de Electrónica, University of Alcalá, 28805, Alcalá de Henares (Madrid), Spain; \\ ${ }^{\mathrm{b}}$ FOCUS S.L., C/ Orellana, 1, $1^{\circ}$ Izq., 28004, Madrid, Spain; \\ ${ }^{\circ}$ EPFL Swiss Federal Institute of Technology, Institute of Electrical Engineering, SCI STI LT, \\ Station 11, CH-1015 Lausanne, Switzerland
}

\begin{abstract}
Chirped-pulse phase-sensitive optical time domain reflectometry has shown a remarkable performance when applied to dynamic measurements of strain and temperature, recently reaching ranges of several kilometers while interrogating the fiber at acoustic frequencies. In this work, its sensitivity, fast response, and high spatial resolution are exploited to implement a proof-of-concept of a selective distributed chemical sensor based on the photothermal effect. The presented scheme is able to perform distributed spectroscopic measurements of acetylene presence along a $10 \mathrm{~m}$-long holey fiber. This potentially gives rise to a new kind of distributed chemical sensors capable of tracking the concentration of chemical species over kilometres.
\end{abstract}

Keywords: Fiber optics sensors, photonic crystal fibers, Rayleigh scattering, photothermal effects, photothermal spectroscopy, time-resolved spectroscopy, chemical analysis

\section{INTRODUCTION}

Over the last years, a great effort has been devoted to the improvement of distributed optical fiber sensors based on phase-sensitive optical time domain reflectometry (TOTDR). These systems are widely described in the literature, and are usually devoted to the (non-metrologic) detection of vibrations along an optical fiber [1]. It has been recently shown that the use of a ФOTDR scheme with chirped pulses can yield single-shot, highly sensitive distributed temperature and strain measurements [2]. In particular, this technique allows monitoring the thermal state of a fiber with high spatial resolution (in the order of meters) and potentially millisecond update rate, with a temperature resolution in the range of millikelvins.

The present work raises one new area of possible application of chirped-pulse TOTDR by taking it as starting point of a distributed chemical sensor based on the photothermal effect. The combination of the salient temperature measurement capabilities of chirped-pulse TOTDR with the fact that an adequate optical signal ( molecules of a substance located in the evanescent field of the fiber core and consequently raise its temperature (photothermal effect) is at the heart of our proposal. In short, our method can be described as follows: an appropriate tunable beam (heating pump) is launched into a holey fiber, where the guided mode shows some overlap with the gas or substance (analyte) to be detected. Simultaneously, the necessary chirped-pulse $\Phi$ OTDR interrogator signal is introduced into the fiber, either from the same end or from the opposite. As the heating pump is absorbed by the analyte, its temperature raises enough to be measured in the adjacent core of the fiber by the chirped-pulse COTDR. Owing to the characteristic spectra of different chemical species, a quasi-simultaneous multi-analyte distributed chemical sensor with spectroscopic specificity could be implemented. Our demonstration could provide a general approach to the development of selective distributed chemical sensors capable of tracking the concentration of several species over kilometers, with high sensitivity and specificity, and in a relatively cheap way.

The relevance of this contribution relies in the qualitative differences between the idea founding our method and other approaches present in the state of art. Until now, the usual way of performing distributed chemical measurements was

*andres.garciaruiz@uah.es; phone +34918856914

25th International Conference on Optical Fiber Sensors, edited by Youngjoo Chung, Wei Jin,

Byoungho Lee, John Canning, Kentaro Nakamura, Libo Yuan, Proc. of SPIE Vol. 10323,

1032361 · C 2017 SPIE · CCC code: 0277-786X/17/\$18 · doi: 10.1117/12.2263210 
based upon an intermediator mechanism, such as a chemical-to-mechanical coating, acting as the transducer that allows to detect and measure the presence of certain substance, by means of the strain produced by the coating in the fiber core [3]. This entails the use of a specific fiber coating making the sensor much more expensive and not adaptable to a wide variety of problems. The mechanism here presented as a proof-of-concept does not depend on a particular fiber for each given analyte, being fully adaptable to multiple substances as long as their absorbance spectrum lie in a region reachable by the pump signal source. When comparing with non-optical means, the advantage in safety is clear when an inflammable or explosive substance is concerned, together with the usual convenience of optical fibers to avoid possible interference issues, or their capability to operate in harsh environments [4]. The lack of a definitive distributed chemical sensor together with the great demand of such a sensor in fields nowadays as important as biosensing, industry, oil and gas extraction, etc. settle the need to explore new and innovative procedures such as the one presented here.

\section{THEORETICAL ESTIMATION OF THE PHOTOTHERMAL EFFECT}

The system we propose here is supported by the use of three important elements: (1) a high-performance distributed thermometer consisting of a TOTDR setup; (2) a suitable holey fibre showing some overlap between the guided mode and the gas to be detected; and (3) a tuneable pumping CW laser signal with a high enough power to produce a temperature increase of the sample gas contained in the holey fibre. Such temperature increase could be detected at the fibre core with the distributed thermometer mentioned first. In our case, the holey fibre shows a $\sim 3 \%$ overlap with the gas contained in the holes, which are filled with acetylene $\left({ }^{12} \mathrm{C}_{2} \mathrm{H}_{2}\right)$. The pump laser can be tuned along the rovibrational spectrum of the acetylene molecule, thus allowing to excite a particular absorption line and discern between a background heating and a contribution due to the sample.

Considering the law of conservation of energy and previous studies made on the thermal behaviour of a structured optical fiber [5], it is known that the thermal distribution is governed by the convection at the interface between the fiber and the surrounding air. Using this as a starting point, some basic calculations can be made to guarantee that the chirpedpulse ФOTDR system will be able to detect the gas heating for reachable pump powers (milliwatts). Thus, we can apply the convection equation, for the expected temperature in the fibre surface:

$$
\Delta T=\frac{P_{\mathrm{abs}}}{h S_{\mathrm{ext}}} .
$$

Here $\Delta T$ represents the difference of temperature between the fiber and the surrounding atmosphere, $P_{\text {abs }}$ is the optical power being absorbed by the analyte, $h$ is the convective coefficient $\left(\sim 15 \mathrm{~W} /\left(\mathrm{m}^{2} \mathrm{~K}\right)\right.$ in our experiment [6]), and $S_{\text {ext }}$ is the external surface of the fiber. Owing to Eq. 1, and considering the absorption in our case, we may expect a temperature increase close to $2 \mathrm{mK}$ per $\mathrm{mW}$ pumped inside the fiber under test (FUT). Although this estimation is enough to confirm the validity of our approach, we are currently working on a more detailed thermal model of photothermal absorption in our holey fiber. There we take into account the distribution of temperature over the whole cross section of the fiber due to heat conduction through its multiple layers, materials and structures.

\section{EXPERIMENTAL SETUP}

The FUT employed (Fig. 1) was a $10 \mathrm{~m}$ long suspended-core photonic crystal fiber (PCF). The core is surrounded by a set of hollow tubes $\sim 5 \mu \mathrm{m}$ in diameter which are arranged in 5 cylindrical layers (holey cladding). An external shell of solid glass cladding completes the structure. Each tube of the fiber is occupied by acetylene $\left({ }^{12} \mathrm{C}_{2} \mathrm{H}_{2}\right)$ at a pressure around 70 mbar. The absorption spectrum of the gas (Fig. 1(a)) presents a series of lines around $1.53 \mu \mathrm{m}$, being the strongest of all (P9), the one used for this experiment.

The probing chirped-pulse $\Phi$ OTDR signal was a $\sim 14 \mathrm{~ns}$ long pulse $(\sim 1.4 \mathrm{~m})$, repeated at a rate of $4 \mathrm{kHz}$. It was produced by a low phase noise telecom oscillator $(1550 \mathrm{~nm})$ followed by a pulsating semiconductor optical amplifier (SOA). The $\mathrm{CW}$ laser diode (LD) was modulated in order to achieve a chirp $\sim 60 \mathrm{MHz}$ wide (giving a $\sim 3 \mathrm{mK}$ resolution), in synchronism with the SOA through sharing the signal generator (SG). The pulse was amplified with an erbium doped fiber amplifier (EDFA), filtered, and injected in the FUT. The backscattered signal was gated by a MEMS variable optical attenuator (VOA) to avoid intense reflection peaks and, after a proper amplifying and filtering stage, it was 
detected with a $1 \mathrm{GHz}$ bandwidth photodetector and digitized at 40 GSPS. The acquired traces were averaged 25 times and the fiber thermal state was recorded every $100 \mathrm{~ms}$.

An amplified, distributed feedback laser (DFB) was used as pumping source. The wavelength of the laser can be electrically tuned with pm resolution. Its power was controlled by a variable attenuator and permanently monitored in a power meter (PM), together with its spectrum at the output of the FUT, where an optical spectrum analyser (OSA) was placed. A wavelength-division multiplexer (WDM) was used to prevent the pump from affecting the DOTDR system.

a)

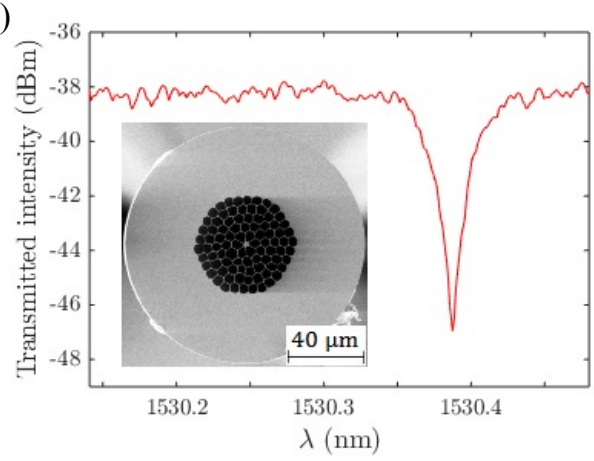

b) TOTDR

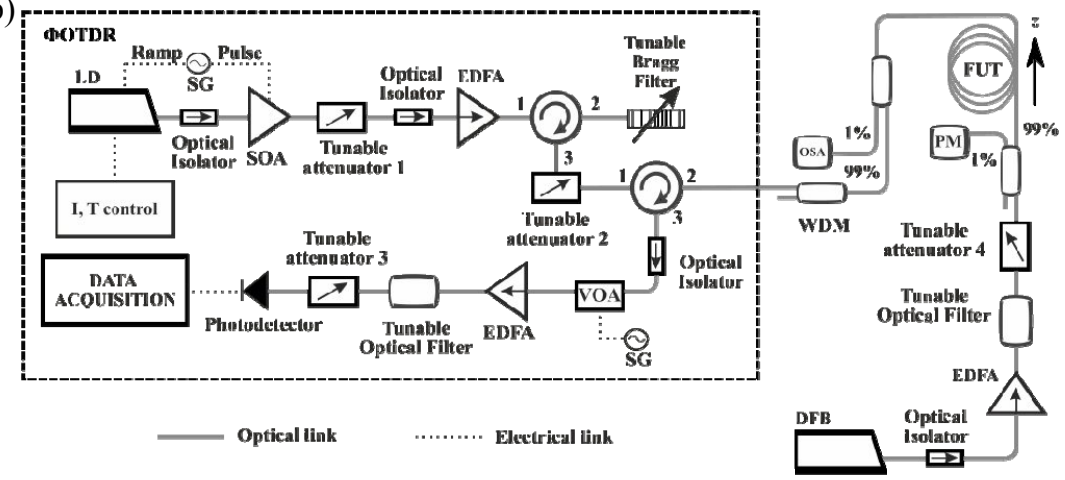

Figure 1. a) P9 line of the acetylene spectrum and fiber cross section. b) Experimental setup employed as distributed chemical sensor. Acronyms are explained within text.

\section{RESULTS}

To distinguish the photothermal contribution from the background temperature changes, the heating pump was frequency-modulated. The wavelength control of the source was driven with a periodic $(50 \mathrm{mHz})$, sinusoidal signal, yielding a variation of the laser central wavelength of up to $7 \mathrm{pm}$ from the P9 line of the acetylene spectrum. This produced a periodic heating at the modulation frequency which can be analysed by means of the Fourier transform in time applied to the local temperature data registered over 30 minutes. The result is shown in Fig 2(a), which shows the spectral content present in the heating map normalized for each point $z$. For each point, we observe the modulation frequency and some of its first harmonics (the origin is taken from the heating pump input). The figure shows the expectable exponential decay of the response due to absorption, which must be taken into account in order to transform the temperature map into gas presence. To make this decay clear, the intensity of the map at the modulation frequency has been taken apart and shown in Fig. 2(b). An exponential fit with the expected pump power loss $(\sim 1.2 \mathrm{~dB} / \mathrm{m})$ is superimposed, showing good agreement. Nevertheless, it is visible that significant departures from the expected tendency appear. These are believed to arise due to the fiber inhomogeneities caused in the fabrication.

a)

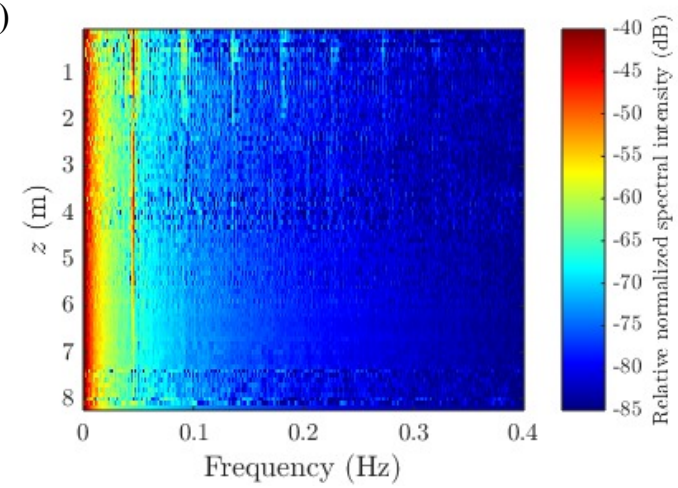

b)

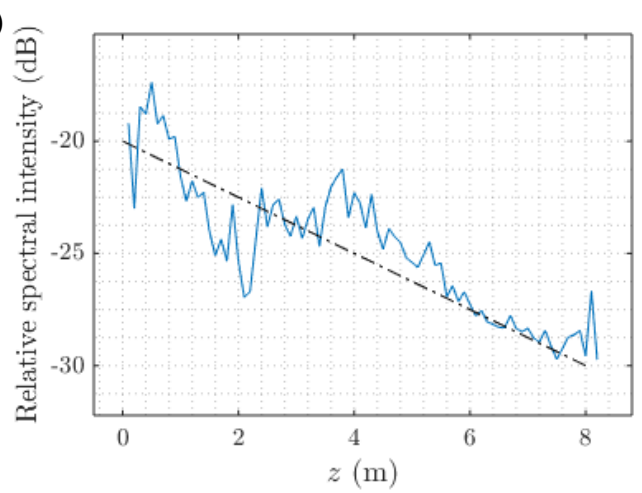

Figure 2. a) Normalized Fourier transform of the temperature evolution over time for each fibre position. b) Curve extracted from 2(a) at the modulation frequency, exhibiting the expected exponential behavior.

Different pump powers were used to test the temporal response of the system when the heating source was set on resonance and suddenly detuned $(-40 \mathrm{pm})$. The resulting transient temperature curves are shown in Fig. 3(a). The average 
of the segments delimited by vertical lines was used to quantify the drop between steady states in order to perform a calibration of the heating due to gas absorption versus pump power. The corresponding plot, together with its linear fit, is shown in Fig. 3(b). As expected, the photothermal heating evolves linearly with the pump power. Further amplification of the pump laser should allow reaching the saturation of the absorption and quantifying the sensitivity limits.

a)

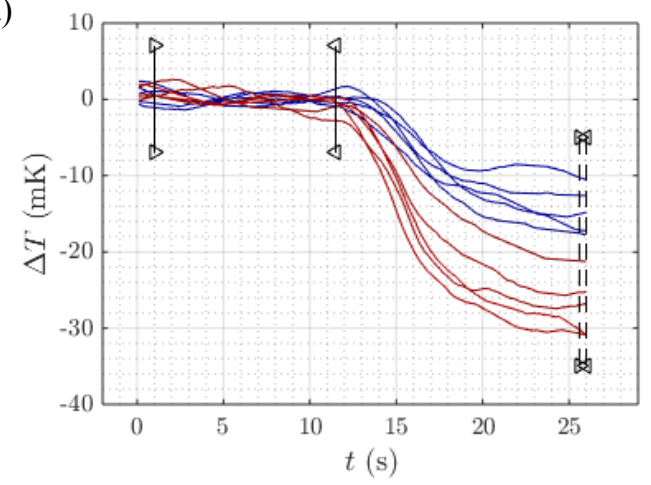

b)

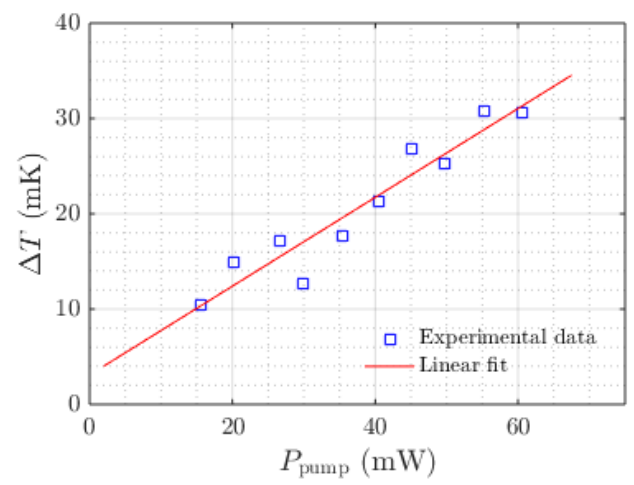

Figure 3. a) Temperature transient states due to the pump going off-resonance for diverse optical heating powers (increasing from bluish to reddish). b) Corresponding temperature drops versus optical heating powers and their linear fit.

\section{CONCLUSIONS}

The present contribution demonstrates the proof of concept of a distributed chemical sensor based on chirped pulsed TOTDR and the photothermal effect. As shown, this technique, which mostly works at telecom wavelengths, avoids the need of special fiber coatings reacting to the presence of a specific analyte, achieving a selective method potentially capable of detecting quasi-simultaneously the presence of several species. The last results shown present a linear relation between the pump power and the heating produced, but increasing the power in order to extend the sensing range may eventually conclude by producing saturated absorption, which may compromise the effective sensing range and the sensitivity of the device. A more detailed study is being carried out to quantify the limits of detection of the technique.

\section{ACKNOWLEDGEMENTS}

This work was supported in part by: the European Research Council through project U-FINE (Grant 307441); the EC Horizon 2020 program; the FINESSE project MSCA-ITN-ETN-722509; the DOMINO Water JPI project, under the WaterWorks2014 co-founded call by EC Horizon 2020 and Spanish MINECO; the Spanish MINECO (projects TEC2013-45265-R and TEC2015-71127-C2-2-R); and the regional program SINFOTON-CM: S2013/MIT-2790. The work of H. F. Martins was supported by EU funding (FP7 ITN ICONE program, gr. \#608099). The work of Juan PastorGraells and S. Martín-López was supported by the Spanish MINECO (FPI and "Ramón y Cajal" contracts, respectively).

\section{REFERENCES}

[1] Martins, H. F., Piote, D., Tejedor, J., Macias-Guarasa, J. et al, "Early detection of pipeline integrity threats using a smart fiber optic surveillance system: the PIT-STOP project," Proc. SPIE 9634, 96347X (2015).

[2] Pastor-Graells, J., Martins, H. F., Garcia-Ruiz, A., Martin-Lopez, S. and Gonzalez-Herraez, M., "Single-shot distributed temperature and strain tracking using direct detection phase-sensitive OTDR with chirped pulses," Opt. Express 24(12), 13121-13133 (2016).

[3] Seitz, W. R., "Chemical sensors based on fiber optics," Anal. Chem. 56(1), 16A-34A (1984).

[4] Calcerrada, M., García-Ruiz, C. and Gonzalez-Herraez, M., "Chemical and biochemical sensing applications of microstructured optical fiber-based systems," Laser Photon. Rev. 9(6), 604-627 (2015).

[5] Limpert, J., Schreiber, T., Liem, A., Nolte, S., Zellmer, H. et al, "Thermo-optical properties of air-clad photonic crystal fiber lasers in high power operation," Opt. Express 11(22), 2982-2990 (2003).

[6] Bergman, T. L., Incropera, F. P. and Lavine, A. S., [Fundamentals of heat and mass transfer], John Wiley \& Sons, 8 (2011). 